\title{
Insulin sensitivity measured by the euglycaemic insulin clamp and proinsulin levels as predictors of stroke in elderly men
}

\author{
B. Wiberg • J. Sundström • B. Zethelius $・$ L. Lind
}

Received: 16 June 2008 / Accepted: 16 September 2008 / Published online: 24 October 2008

(C) Springer-Verlag 2008

\begin{abstract}
Aims/hypothesis Our aim was to investigate the predictive power of a panel of variables in glucose and insulin metabolism for the incidence of stroke or transient ischaemic attacks (TIA). We hypothesised that proinsulin and insulin resistance contributes to an increase of risk for fatal and non-fatal stroke/TIA, independently of diabetes and established risk factors.

Methods The study is based on the Uppsala Longitudinal Study of Adult Men cohort. The examinations were performed at age 70 years.

Results In 1,151 men free from stroke at baseline, 150 developed stroke or TIA during a median follow-up of 8.8 years. In unadjusted Cox proportional hazards analyses, a $1 \mathrm{SD}$ increase of a predictor variable was associated with an increased risk for stroke/TIA, e.g. plasma insulin (HR $1.19,95 \%$ CI 1.01-1.40), fasting intact proinsulin (HR $1.28,95 \%$ CI 1.09-1.49); whereas a 1 SD increase in insulin sensitivity measured by the euglycaemic insulin clamp method decreased the risk for stroke/TIA (HR 0.81, 95\% CI 0.68-0.96). The predictive values of fasting intact proinsulin and insulin sensitivity endured but not that of plasma insulin when adjusting for diabetes. In models adjusting for diabetes, hypertension, atrial fibrillation, electrocardiographic left ventricular hypertrophy, serum cholesterol and smoking, proinsulin remained as a signif-
\end{abstract}

B. Wiberg $(\varangle) \cdot$ B. Zethelius

Department of Public Health and

Caring Sciences/Geriatrics, Uppsala University,

Uppsala Science Park,

SE-751 85 Uppsala, Sweden

e-mail: bernice.wiberg@akademiska.se

J. Sundström $\cdot$ L. Lind

Department of Medical Sciences, Uppsala University,

Uppsala, Sweden icant predictor of later stroke/TIA (HR 1.22, 95\% CI 1.001.48) whereas clamp insulin sensitivity did not (HR 0.87 , 95\% CI 0.71-1.07).

Conclusions/interpretation Fasting intact proinsulin level and insulin sensitivity at clamp predicted subsequent fatal and non-fatal stroke/TIA, independently of diabetes in elderly men whereas fasting insulin did not.

Keywords Clamp · Insulin resistance · Proinsulin .

Risk factors $\cdot$ Stroke

$\begin{array}{ll}\text { Abbreviations } \\ \text { BI } & \text { brain infarction } \\ \text { BW } & \text { body weight } \\ \text { CDR } & \text { Cause-of-Death Register } \\ \text { ICH } & \text { intracerebral haemorrhage } \\ \text { LVH } & \text { left ventricular hypertrophy } \\ M & \text { glucose disposal rate } \\ M / I & \text { insulin sensitivity index } \\ \text { PYAR } & \text { person-years at risk } \\ \text { SAH } & \text { subarachnoidal haemorrhage } \\ \text { SHDR } & \text { Swedish Hospital Discharge Record } \\ \text { TIA } & \text { transient ischaemic attack }\end{array}$

\section{Introduction}

Stroke is a major cause of morbidity and mortality. For this reason, the identification of modifiable risk factors for stroke is of great importance. The discovery of new glucose and insulin metabolism cardiovascular risk factors in recent years has prompted a more detailed characterisation of these variables as stroke/transient ischaemic attack (TIA) predictors. 
Insulin resistance is common in stroke/TIA patients [1]. A relation between elevated fasting proinsulin and stroke has previously been reported in a case-control study [2] and in a population-based cohort study [3]. However, longitudinal studies that include a detailed characterisation of glucose and insulin metabolism predictors of stroke, are lacking.

We hypothesised that proinsulin and insulin resistance contribute to an increased risk for fatal and non-fatal stroke/ TIA, independently of diabetes and established risk factors. Accordingly, we investigated the predictive power of baseline glucose and insulin metabolism variables for incident stroke/TIA and subgroups of brain infarction (BI) and intracerebral haemorrhage (ICH) in a community-based sample of elderly men followed for a median of 8.8 years.

\section{Methods}

\section{Study population}

The study is based on the Uppsala Longitudinal Study of Adult Men cohort (http://www.pubcare.uu.se/ULSAM), a health survey focusing on identifying metabolic risk factors for cardiovascular disease, to which all 50-year-old men living in Uppsala, Sweden, in 1970-1974 were invited. Of these, 2,322 (82\%) agreed to be included in the investigation. A reinvestigation was performed 20 years later and this comprises the baseline of the present study. Of the 1,681 available men invited to this reinvestigation 1,221 (73\%) attended. Seventy men were excluded because of a history of stroke or TIA before the baseline examination, giving 1,151 participants in this study. To maximise statistical power, only participants with a missing covariate needed for that particular model were excluded from the analyses. All participants gave written consent and the Ethics Committee of Uppsala University approved the study.

\section{Baseline examinations}

These examinations have previously been described in detail [4]. The examinations were performed when the participants were 70 years old and included a medical questionnaire concerning previous diseases and medical treatment. The participants underwent a physical examination that included anthropometric measurements. Blood pressures were measured under standardised conditions. Hypertension prevalence was defined as a supine blood pressure of 140/90 $\mathrm{mmHg}$ or greater and/or treatment with anti-hypertensive drugs for hypertension. Blood samples were drawn in the morning after an overnight fast. A standard 12-lead ECG was recorded. The revised Minnesota code was used for classification [5, 6], and left ventricular hypertrophy (LVH) was defined as high amplitude R-waves together with a left ventricular strain pattern. Diabetes was defined according to the 1997 American Diabetes Association criteria [7].

Cholesterol concentrations were measured in serum by enzymatic techniques using the IL rest cholesterol Trinder's method for use in a Monarch apparatus (Instrumentation Laboratories, Lexington, KT, USA). Particles of HDLcholesterol were separated by precipitation with magnesium chloride/phosphotungstate. During the clamp investigation, a nurse or a technician asked the participant the question: 'Do you smoke?'

Glucose- and insulin-related variables

Fasting concentrations of intact proinsulin and 32-33 split proinsulin were measured using a two-site immunometric assay technique [8], and specific insulin was measured by the Access Immunoassay System (Beckman Coulter, Chaska, MN, USA), which uses a chemiluminescent immunoenzymatic assay. These analyses were carried out at the Department of Clinical Biochemistry, Addenbrooke's Hospital, Cambridge, UK.

Plasma glucose in samples from the OGTT was measured by the glucose dehydrogenase method (Gluc-DH, Merck, Darmstadt, Germany).

The euglycaemic insulin clamp was used to estimate in vivo sensitivity to insulin. Plasma insulin was measured by an immunoenzymatic assay (Enzymmun, Boehringer Mannheim, Mannheim, Germany) performed in an ES300 automatic analyser (Boehringer Mannheim).

To achieve complete suppression of hepatic glucose output, insulin was infused at a constant rate of 56 instead of $40 \mathrm{mU} \mathrm{min}{ }^{-1} \times \mathrm{m}^{-2}$ ). The level of plasma glucose was maintained by measuring the plasma glucose every $5 \mathrm{~min}$ and adjusting the rate of infusion of a $20 \%$ (wt/vol.) glucose solution accordingly. The target plasma glucose level was $5.1 \mathrm{mmol} / \mathrm{l}$. The total amount of glucose infused serves as a measure of the participant's sensitivity to the prevailing plasma insulin concentrations.

The glucose disposal rate $(M)$ was calculated as the amount of glucose taken up during the last $60 \mathrm{~min}$ of the $120 \mathrm{~min}$ insulin clamp. The insulin sensitivity index $(\mathrm{M} / \mathrm{I})$ was used as a measure of the tissue sensitivity to insulin expressed per unit insulin and was calculated by dividing $M$ by the mean insulin concentration $(I)$ during the same period of the clamp. Consequently, $M / I$ represents the amount of glucose metabolised per unit of plasma insulin.

\section{Follow-up}

Participants were followed from the baseline investigation at age 70 years (August 1991 to May 1995) until 31 
December 2002. The participants had a median follow-up time of 8.8 years (range 0.01 to 11.4 years), contributing to 9,395 person-years at risk (PYAR).

\section{Endpoints}

To determine the occurrence of endpoints, two registers were used, the Swedish Hospital Discharge Record (SHDR) and the Cause-of-Death Register (CDR). The endpoints investigated in the study were, in order of priority (http:// www.who.int/classifications/icd/en/):

1. Fatal or non-fatal stroke or TIA (ICD-9 codes $430-432$ and 434-436, ICD-10 codes I60-I64, I66 and G45);

2. Fatal or non-fatal BI (ICD-9 code 434 , ICD-10 code I63);

3. Fatal or non-fatal ICH (ICD-9 codes $431-432$, ICD-10 codes I61-I62).

Some participants experienced more than one of the stipulated endpoints during the follow-up period and could appear in more than one endpoint analysis. Only the first occurrence of the endpoint studied in a particular model merited censoring (see Fig. 1).

\section{Statistical analyses}

Distributions were tested for normality by the ShapiroWilk $W$ test and logarithmic transformation was performed when $W$ was $<0.95$. The prognostic value of a $1 \mathrm{SD}$ increase in the continuous variables (which is essentially

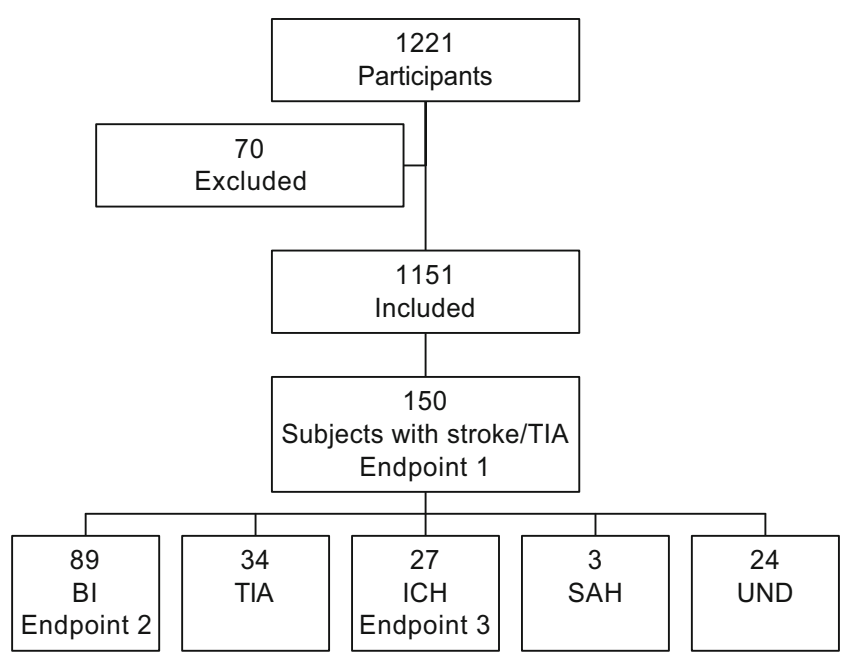

Fig. 1 Description of endpoints. Endpoint 1: all participants with fatal and non-fatal stroke/TIA. Endpoint 2: all participants with fatal and non-fatal BI or TIA. Endpoint 3: all participants with fatal or non-fatal ICH. Only the first occurrence of the endpoint studied in a particular model merited censoring. Some participants experienced a first occurrence of more than one endpoint, and were therefore included in more than one endpoint analysis. UND, undetermined given in the 'no stroke or TIA' group in Table 1), or transfer from one level to another of the dichotomous variables, was investigated with Cox proportional HRs. Nelson-Aalen curves were used to confirm proportionality of hazards and to describe risk over time graphically. We examined three sets of models in a hierarchical fashion: unadjusted; adjusted for diabetes at baseline; and adjusted for diabetes plus other established risk factors for stroke (hypertension, atrial fibrillation, ECG-LVH, serum cholesterol and smoking) determined at baseline. Two-tailed 95\% CI and $p$ values were given. The statistical software package Stata 10.0 (Stata Corporation, College Station, TX, USA) was used.

\section{Results}

Baseline characteristics are described in Table 1. During follow-up, 150 cases of stroke/TIA occurred (incidence rate 16/1,000 PYAR); of which 89 were BI and 27 were ICH (incidence rates 9.3/1,000 and 2.7/1,000 PYAR, respectively). There were 34 TIA, three subarachnoidal haemorrhage (SAH) and 24 were undetermined types. During the followup period 275 men died and stroke was the cause in 17 cases.

Baseline clinical characteristics and standardised crude HRs for the outcomes first stroke/TIA, BI and ICH are presented in Table 2 for traditional risk factors and in Table 3 for insulin variables. In the Cox proportional hazards models, hypertension, ECG-LVH, atrial fibrillation, diabetes, plasma insulin, intact and split 32-33 proinsulin, and the $M / I$ ratio were all associated with a subsequent stroke/TIA, whereas fasting plasma glucose, BMI, waist circumference, serum cholesterol and current smoking were not. A similar picture emerged when BI was used as the outcome variable, but none of the glucose and insulin variables were significant predictors of ICH. The predictive values for stroke/TIA of intact proinsulin and the $M / I$ were preserved when adjustment was made for diabetes, but not for plasma insulin or split 32-33 proinsulin.

In multivariable models with additional adjustments for hypertension, atrial fibrillation, ECG-LVH, serum cholesterol and current smoking (Table 2), intact proinsulin was retained as a significant independent predictor of stroke/ TIA, but not of BI (Table 3). No significant risk was related to $M / I$. We were unable to detect significant associations with $\mathrm{ICH}$.

If $M$ was chosen instead of $M / I$, essentially the same picture emerged both when adjusting for diabetes and in multivariable models.

The longitudinal relation of $M / I$ and intact proinsulin level to the incidence of stroke/TIA is presented in Fig. 2. No apparent deviations from linearity were observed when 
Table 1 Clinical characteristics at baseline

\begin{tabular}{|c|c|c|c|c|}
\hline Clinical characteristic & No stroke or TIA $(n=1001)$ & Stroke or TIA $(n=150)$ & $\mathrm{BI}(n=89)$ & $\mathrm{ICH}(n=27)$ \\
\hline Body mass index $\left(\mathrm{kg} / \mathrm{m}^{2}\right)$ & $26.2(3.4)$ & $26.5(3.5)$ & $26.6(3.2)$ & $25.7(3.1)$ \\
\hline Waist circumference $(\mathrm{cm})$ & $94.6(9.6)$ & $95.4(9.5)$ & $95.9(9.3)$ & $93.8(8.5)$ \\
\hline Hypertension & $731(73)$ & $126(84)$ & $78(88)$ & $23(85)$ \\
\hline ECG-LVH & $58(6)$ & $21(16)$ & $16(21)$ & $4(19)$ \\
\hline Atrial fibrillation & $31(3)$ & $16(12)$ & $12(14)$ & $3(13)$ \\
\hline Diabetes & $102(10)$ & $22(15)$ & $14(16)$ & $3(11)$ \\
\hline P-glucose (mmol/l) & $5.74(1.41)$ & $5.96(1.88)$ & $6.08(2.03)$ & $5.63(0.94)$ \\
\hline P-insulin $(\mathrm{pmol} / \mathrm{l})$ & $51.6(45.5)$ & $59.2(47.7)$ & $64.1(52.5)$ & $43.2(19.6)$ \\
\hline P-intact proinsulin (pmol/1) & $8.40(8.9)$ & $9.25(6.6)$ & $9.35(6.5)$ & $7.83(4.7)$ \\
\hline P-32-33 split proinsulin $(\mathrm{pmol} / \mathrm{l})$ & $10.91(12.4)$ & $11.60(10.2)$ & $12.15(11.0)$ & $9.37(5.5)$ \\
\hline$M\left(\mu \mathrm{mol}[\mathrm{kg} \mathrm{BW}]^{-1} \min ^{-1}\right)$ & $4.70(2.30)$ & $4.28(2.19)$ & $4.15(2.11)$ & $4.76(2.33)$ \\
\hline$M / I\left(\mu \mathrm{mol}[\mathrm{kg} \mathrm{BW}]^{-1} \min ^{-1}[\mathrm{mU} / 1]^{-1} \times 100\right)$ & $4.61(2.26)$ & $4.20(2.15)$ & $4.07(2.07)$ & $4.67(2.29)$ \\
\hline S-total cholesterol (mmol/l) & $5.8(1.0)$ & $5.9(1.1)$ & $6.0(1.1)$ & $5.6(1.1)$ \\
\hline S-HDL-cholesterol (mmol/l) & $1.29(0.35)$ & $1.26(0.33)$ & $1.27(0.34)$ & $1.24(0.31)$ \\
\hline Current cigarette smoking & $199(21)$ & $31(21)$ & $20(23)$ & $5(19)$ \\
\hline
\end{tabular}

Data are means (standard deviations), or numbers of individuals (per cent)

$\mathrm{P}$, plasma; S, serum

investigating incidence rate by quartiles of the independent variables.

\section{Discussion}

In this community-based sample of elderly men free of stroke/TIA at baseline, both a low insulin sensitivity index derived from the euglycaemic insulin clamp [9] and elevated fasting intact proinsulin levels, predicted subsequent stroke/TIA over a follow-up period of 8.8 years independently of diabetes.

The present finding that elevated intact proinsulin might be a stronger predictor of stroke/TIA than is true insulin in this elderly population, extends our previous observations concerning the predictive power of proinsulin for coronary heart disease [10] and heart failure [10]. Our results may indicate that the risk of stroke/TIA is increased in the long subclinical phase of impaired glucose regulation that precedes clinically manifest diabetes because an elevated proinsulin level is a predictor of diabetes development [11]. Proinsulin is a powerful predictor of stroke/TIA and was, furthermore, a significant predictor independently of diabetes and other established risk factors for stroke.

Defective insulin action as assessed by the euglycaemic insulin clamp is associated with a well-established cluster of abnormalities, consisting of type 2 diabetes mellitus, hypertension, dyslipidaemia and abdominal obesity, elevat-

Table 2 Unadjusted HR for the relationships of baseline clinical variables to stroke/TIA, BI and ICH outcomes vs participants without stroke/TIA $(n=1001)$

\begin{tabular}{|c|c|c|c|c|c|c|}
\hline \multirow[t]{2}{*}{ Clinical characteristic } & \multicolumn{2}{|c|}{ Stroke or TIA $(n=150)$} & \multicolumn{2}{|l|}{$\mathrm{BI}(n=89)$} & \multicolumn{2}{|l|}{$\mathrm{ICH}(n=27)$} \\
\hline & HR $(95 \% \mathrm{CI})$ & $p$ value & $\mathrm{HR}(95 \% \mathrm{CI})$ & $p$ value & HR $(95 \% \mathrm{CI})$ & $p$ value \\
\hline Body mass index & $1.09(0.93-1.28)$ & 0.272 & $1.12(0.91-1.37)$ & 0.283 & $0.84(0.56-1.26)$ & 0.410 \\
\hline Waist circumference & $1.10(0.93-1.29)$ & 0.264 & $1.16(0.94-1.43)$ & 0.164 & $0.92(0.62-1.36)$ & 0.671 \\
\hline Hypertension & $1.88(1.22-2.92)$ & 0.004 & $2.51(1.33-4.72)$ & 0.004 & $2.00(0.69-5.79)$ & 0.200 \\
\hline ECG-LVH & $2.68(1.68-4.27)$ & $<0.001$ & $3.66(2.11-6.35)$ & $<0.001$ & $3.09(1.04-9.18)$ & 0.042 \\
\hline Atrial fibrillation & $3.64(2.16-6.15)$ & $<0.001$ & $4.77(2.58-8.81)$ & $<0.001$ & $3.66(1.09-12.35)$ & $<0.001$ \\
\hline Diabetes & $1.61(1.02-2.53)$ & 0.040 & $1.74(0.99-3.10)$ & 0.055 & $1.09(0.33-3.62)$ & 0.887 \\
\hline P-glucose & $1.14(0.99-1.32)$ & 0.064 & $1.28(1.10-1.50)$ & 0.002 & $0.94(0.62-1.41)$ & 0.766 \\
\hline S-cholesterol & $1.06(0.90-1.25)$ & 0.442 & $1.20(0.98-1.48)$ & 0.078 & $0.78(0.53-1.15)$ & 0.206 \\
\hline Current cigarette smoking & $1.10(0.74-1.64)$ & 0.623 & $1.23(0.75-2.03)$ & 0.413 & $0.96(0.36-2.56)$ & 0.943 \\
\hline
\end{tabular}

Data are Cox proportional hazard ratios $(95 \%$ CIs) for 1 SD increase in the continuous, or transfer from one level to another of the dichotomous variables

Diabetes is defined according to the 1997 American Diabetes Association criteria [7]

P, plasma; S, serum 
Table 3 HRs for the relationships of baseline clinical variables to stroke/TIA, BI and ICH outcomes vs participants without stroke/TIA ( $n=1001)$

\begin{tabular}{|c|c|c|c|}
\hline Clinical characteristic & Unadjusted model & Models adjusted for diabetes & Multi-variable adjusted model \\
\hline \multicolumn{4}{|l|}{ Stroke or TIA $(n=150)$} \\
\hline P-insulin & $1.19(1.01-1.40)$ & $1.16(0.98-1.37)$ & $1.10(0.92-1.32)$ \\
\hline$p$ value & 0.036 & 0.086 & 0.287 \\
\hline S-intact proinsulin & $1.28(1.09-1.49)$ & $1.25(1.04-1.49)$ & $1.22(1.00-1.48)$ \\
\hline$p$ value & 0.002 & 0.015 & 0.051 \\
\hline S-32-33 split proinsulin & $1.20(1.02-1.42)$ & $1.16(0.97-1.38)$ & $1.17(0.97-1.43)$ \\
\hline$p$ value & 0.026 & 0.105 & 0.051 \\
\hline$M / I$ & $0.81(0.68-0.96)$ & $0.83(0.69-1.00)$ & $0.87(0.71-1.07)$ \\
\hline$p$ value & 0.017 & 0.054 & 0.107 \\
\hline \multicolumn{4}{|l|}{$\mathrm{BI}(n=89)$} \\
\hline P-insulin & $1.33(1.08-1.63)$ & $1.27(1.03-1.57)$ & $1.14(0.91-1.43)$ \\
\hline$p$ value & 0.007 & 0.026 & 0.251 \\
\hline S-intact proinsulin & $1.31(1.07-1.60)$ & $1.23(0.98-1.56)$ & $1.18(0.91-1.52)$ \\
\hline$p$ value & 0.009 & 0.075 & 0.201 \\
\hline S-32-33 split proinsulin & $1.25(1.01-1.55)$ & $1.17(0.93-1.48)$ & $1.15(0.89-1.48)$ \\
\hline$p$ value & 0.035 & 0.174 & 0.282 \\
\hline$M / I$ & $0.76(0.60-0.96)$ & $0.78(0.60-1.00)$ & $0.81(0.62-1.07)$ \\
\hline$p$ value & 0.020 & 0.049 & 0.146 \\
\hline \multicolumn{4}{|l|}{$\mathrm{ICH}(n=27)$} \\
\hline P-insulin & $0.84(0.56-1.25)$ & $0.84(0.56-1.27)$ & $0.95(0.60-1.50)$ \\
\hline$p$ value & 0.389 & 0.420 & 0.831 \\
\hline S-intact proinsulin & $1.04(0.70-1.55)$ & $1.09(0.70-1.70)$ & $1.28(0.78-2.09)$ \\
\hline$p$ value & 0.835 & 0.696 & 0.322 \\
\hline S-32-33 split proinsulin & $0.99(0.66-1.47)$ & $1.01(0.66-1.56)$ & $1.31(0.80-2.14)$ \\
\hline$p$ value & 0.950 & 0.948 & 0.284 \\
\hline$M / I$ & $1.03(0.71-1.51)$ & $1.05(0.70-1.57)$ & $1.15(0.73-1.80)$ \\
\hline$p$ value & 0.865 & 0.804 & 0.552 \\
\hline
\end{tabular}

Data are Cox proportional hazard ratios (95\% CIs) for a $1 \mathrm{SD}$ increase in the continuous, or transfer from one level to another of the dichotomous variables. 'Multivariable adjusted models' adjusted for; hypertension, diabetes, atrial fibrillation, ECG-LVH, serum cholesterol and smoking $\mathrm{P}$, plasma; $\mathrm{S}$, serum

ed plasma triacylglycerol, decreased HDL-cholesterol and microalbuminuria, which together constitute the insulin resistance syndrome [12]. The present study showed insulin resistance, as evaluated by this gold standard technique, to be a predictor of stroke/TIA independently of diabetes. However, when additional adjustments were made for all established risk factors for stroke, $M / I$ was attenuated and was no longer significant $(p=0.107)$. This further emphasises the difficulties in separating the different components of the metabolic syndrome in terms of independent risk predictors.

There are numerous possible explanations for the observed relation of proinsulin and insulin resistance to the incidence of stroke/TIA. First, high plasma proinsulin concentrations are associated with coronary artery atherosclerosis [13] and with an increased intima-media thickness in the common carotid artery [14]. In clinical trials greatly increased human proinsulin was found to be associated with a greater increase in cardiovascular events in comparison with elevated human insulin, suggesting that thromboembolic mechanisms had been activated [15]. Second, elevat- ed plasma non-esterified fatty acids are known to aggravate the insulin-resistant state both in skeletal muscle and in the liver [16], and also to have direct vascular effects such as promotion of endothelial dysfunction [17] and elevation of blood pressure [18], the most well-established risk factor for stroke/TIA. Third, insulin resistance is related to an increased pressor response to angiotensin II [19] and has been found to increase the stimulating effects of angiotensin II on cellular hypertrophy and collagen production [20] in individuals with hypertension. Fourth, a deranged microcirculation with vascular hypertrophy and rarefaction has been proposed as a central pathological feature in insulin resistance [21].

Strengths of the present investigation include the large community-based sample, the long follow-up, the nonexistent loss to follow-up, and the comprehensive characterisation of the included variables. To our knowledge, the ULSAM cohort is the largest single-centre population-based cohort that has been examined with the gold standard for measurement of insulin resistance, the euglycaemic insulin clamp method. The study sample is also representative of the 
a

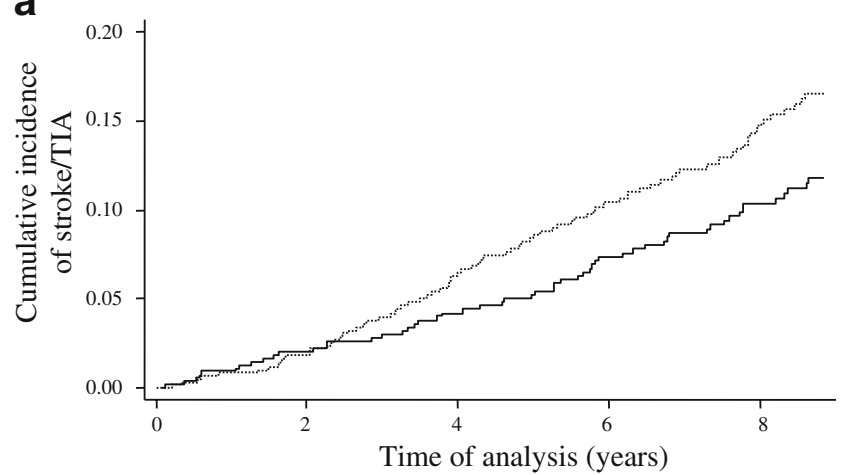

b

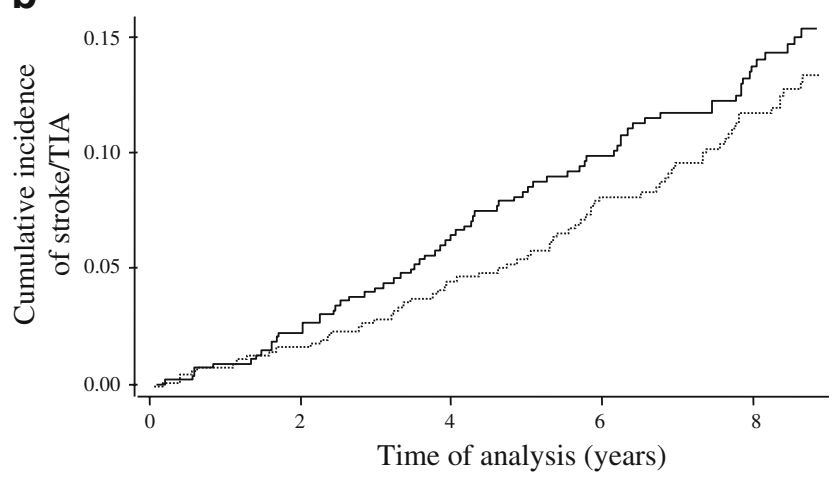

Fig. 2 a Cumulative incidence of stroke/TIA by proinsulin at or above vs below median in unadjusted models. Continuous line, proinsulin below median; dotted line, proinsulin at or above median. b Cumulative incidence of stroke/TIA by $M / I$ ratio at or above vs below median in unadjusted models. Continuous line, $M / I$ below median; dotted line, $M / I$ at or above median

general population in Sweden regarding stroke incidence, according to data published by the Swedish National Board of Health and Welfare (http://www.socialstyrelsen.se/).

As we only examined men of similar age with the same ethnic background, the results may have limited generalisability to women and to other age groups and ethnic groups. The present cohort has been re-examined several times since baseline and risk factors have been treated and the participants may therefore have been healthier during follow-up than the average man. Therefore, the associations found in this study may have driven to the null-hypothesis. It is also an elderly cohort and therefore those with more usual pathways concerning risk factors may already have been weeded out by the time they have reached the age of 70 years.

Another limitation is that follow-up data concerning medication, including hypoglycaemic agents and antiplatelet therapy, over time is not available. Other limitations of the study include possible misclassification of stroke, although the accuracy of the SHDR and the CDR has been shown to be high regarding the stroke diagnosis [22].

To conclude, concentrations of plasma intact proinsulin and insulin sensitivity index measured by the euglycaemic insulin clamp [9], at age 70 years predict subsequent fatal and non-fatal stroke/TIA, independently of diabetes in a community-based sample of men followed for up to 12 years. Intact proinsulin also preserves its predictive value after adjustment for other traditional risk factors. No other variable in the investigated panel including true insulin and plasma glucose, usually measured on a routine basis in medical care, has the same predictive value. Proinsulin is suggested to be a surrogate marker for insulin sensitivity. Further studies are needed to evaluate proinsulin as a surrogate marker in future clinical studies.

Acknowledgements This study was kindly supported by grants from the Medical Faculty at Uppsala University, Stroke Riksförbundet, Swedish Heart Lung Foundation, Ernfors Family Foundation, Thurin Foundation and Selander Foundation. We thank A. Terént (Department of Medical Sciences, Uppsala University) for valuable comments on the manuscript.

Duality of interest The authors declare that there is no duality of interest associated with this manuscript.

\section{References}

1. Kernan WN, Inzucchi SE, Viscoli CM et al (2003) Impaired insulin sensitivity among nondiabetic patients with a recent TIA or ischemic stroke. Neurology 60:1447-1451

2. Lindahl B, Dinesen B, Eliasson M, Roder M, Hallmans G, Stegmayr B (2000) High proinsulin levels precede first-ever stroke in a nondiabetic population. Stroke 31:2936-2941

3. Wiberg B, Sundstrom J, Arnlov J et al (2006) Metabolic risk factors for stroke and transient ischemic attacks in middle-aged men: a community-based study with long-term follow-up. Stroke 37:2898-2903

4. Zethelius B, Hales CN, Lithell HO, Berne C (2004) Insulin resistance, impaired early insulin response, and insulin propeptides as predictors of the development of type 2 diabetes: a population-based, 7-year follow-up study in 70-year-old men. Diabetes Care 27:1433-1438

5. Prineas R, Crow R, Blackburn H (1982) The Minnesota code manual of electrocardiographic findings standards and procedures for measurement and classification. John Wright-PSG, Inc., Littleton, MA, USA

6. Blackburn H, Keys A, Simonson E, Rautaharju P, Punsar S (1960) The electrocardiogram in population studies. A classification system. Circulation 21:1160-1175

7. ADA (1997) American Diabetes Association: clinical practice recommendations 1997. Diabetes Care 20(Suppl 1):S1-S70

8. Sobey WJ, Beer SF, Carrington CA et al (1989) Sensitive and specific two-site immunoradiometric assays for human insulin, proinsulin, 65-66 split and 32-33 split proinsulins. Biochem J 260:535-541

9. DeFronzo RA, Tobin JD, Andres R (1979) Glucose clamp technique: a method for quantifying insulin secretion and resistance. Am J Physiol 237:E214-E223

10. Ingelsson E, Arnlov J, Sundstrom J, Zethelius B, Vessby B, Lind L (2005) Novel metabolic risk factors for heart failure. J Am Coll Cardiol 46:2054-2060

11. Zethelius B, Byberg L, Hales CN, Lithell H, Berne C (2003) Proinsulin and acute insulin response independently predict type 2 diabetes mellitus in men-report from 27 years of follow-up study. Diabetologia 46:20-26 
12. Reaven GM, Laws A (1994) Insulin resistance, compensatory hyperinsulinaemia, and coronary heart disease. Diabetologia 37:948-952

13. Bavenholm P, Proudler A, Tornvall $P$ et al (1995) Insulin, intact and split proinsulin, and coronary artery disease in young men. Circulation 92:1422-1429

14. Haffner SM, D'Agostino R, Mykkanen L et al (1998) Proinsulin and insulin concentrations in relation to carotid wall thickness: Insulin Resistance Atherosclerosis Study. Stroke 29:1498-1503

15. Galloway JA, Hooper SA, Spradlin CT et al (1992) Biosynthetic human proinsulin. Review of chemistry, in vitro and in vivo receptor binding, animal and human pharmacology studies, and clinical trial experience. Diabetes Care 15:666-692

16. Boden G (1997) Role of fatty acids in the pathogenesis of insulin resistance and NIDDM. Diabetes 46:3-10

17. Steinberg HO, Chaker H, Leaming R, Johnson A, Brechtel G, Baron AD (1996) Obesity/insulin resistance is associated with endothelial dysfunction. Implications for the syndrome of insulin resistance. J Clin Invest 97:2601-2610
18. Salonen JT, Lakka TA, Lakka HM, Valkonen VP, Everson SA, Kaplan GA (1998) Hyperinsulinemia is associated with the incidence of hypertension and dyslipidemia in middle-aged men. Diabetes 47:270-275

19. Gaboury CL, Simonson DC, Seely EW, Hollenberg NK, Williams GH (1994) Relation of pressor responsiveness to angiotensin II and insulin resistance in hypertension. J Clin Invest 94:22952300

20. Sartori M, Ceolotto G, Papparella I et al (2004) Effects of angiotensin II and insulin on ERK1/2 activation in fibroblasts from hypertensive patients. Am J Hypertens 17:604-610

21. Lithell H, Lindgarde F, Hellsing K et al (1981) Body weight, skeletal muscle morphology, and enzyme activities in relation to fasting serum insulin concentration and glucose tolerance in 48year-old men. Diabetes 30:19-25

22. Merlo J, Lindblad U, Pessah-Rasmussen H et al (2000) Comparison of different procedures to identify probable cases of myocardial infarction and stroke in two Swedish prospective cohort studies using local and national routine registers. Eur J Epidemiol 16:235-243 